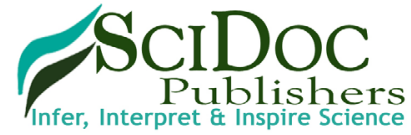

\section{Visual Field Loss, Quality of Life and Ocular Biometric Parameters: A Cross Sectional Study of Taiwanese Adults}

\author{
Research Article
}

Hsieh $\mathrm{MH}^{1}$, Lin $\mathrm{JC}^{1,2^{*}}$

${ }^{1}$ Department of Ophthalmology, Taipei City Hospital, Heping Fuyou Branch, Taipei, Taiwan.

${ }^{2}$ Graduate Institute of Neuroscience, National Chengchi University, Taipei, Taiwan.

\title{
Abstract
}

Purpose: To evaluate the severity of visual field loss in Taiwanese adults and assess the associations between visual field loss detected by frequency doubling technology (FDT) perimetry and vision-related quality of life (QoL) in Taiwanese adults. Methods: A total of 206 eyes from 206 subjects were included. Each subject was examined using FDT (N-30 threshold test) perimetry, optical coherence tomography (OCT), noncontact tonometry, cycloplegic refractometry, and National Eye Institute Visual Functioning Questionnaire 25 items (NEI VFQ-25). Results of FDT tests were classified into four groups (normal, early, moderate, and severe defects) using FDT probability map analysis. OCT parameters included central cornea thickness (CCT), thickness of retinal nerve fiber layer (RNFL), ganglion cell complex (GCC), focal loss volume (FLV), and global loss volume (GLV) of GCC. The global score, subscales of near and distance activities of VFQ were calculated. Linear regression analysis was used to analyze the factors associated with visual field loss. Kruskal-Wallis analysis was used to examine the relationship between FDT results and vision-related QoL.

Results: Among the 206 subjects, 72 were normal. The numbers of participants having early, moderate, and severe visual field loss were 101, 27, and 6. Moderate visual field defect was associated with CCT, GLV, GCC, and SE when compared to the subjects with normal response. Kruskal-Wallis analysis indicated that those with moderate visual field loss determined by FDT had worse vision related QoL and more difficulties in distance activities of VFQ $(p=0.01)$ than people with normal response.

Conclusion: Moderate visual field loss detected by FDT was associated with vision-related QoL in young adults. Further study is warranted to determine the potential of visual field loss as a predicting factor for vision loss related QoL.

Keywords: Visual Field Loss; Quality of Life; Ocular Biometric Parameter; VFQ25.

\section{Introduction}

Visual field loss is usually detected in young adults in clinical practice. The major concern of the functional loss of vision is the consequent disability and decreased vision-related quality of life (QoL). The relationship between visual field loss and QoL has a prominent impact on therapeutic decisions.

Frequency doubling technology (FDT) perimetry, with a similar sensitivity to visual field loss and advantages of shorter test duration and lower cost than standard automated perimetry (SAP) [1], has become a popular screening strategy in clinical settings in Taiwan. Given its lower variability compared to SAP, FDT provides a convenient approach to effectively detect visual field loss in a sequence of tests for adults, and it may detect progressive visual field loss not apparent on SAP [2]. We are interested in determining the association between FDT detected and confirmed visual field loss with a decrease in vision-related QoL. Thus, the purpose of present study was to evaluate the severity of visual field defects according to the results of FDT perimetry and assess the association with QoL using data measured by the National Eye Institute Visual Function Questionnaire (NEI VFQ-25) in young adults in a cross-sectional setting.

\section{Methods}

A cross-sectional study was designed and conducted in a regional hospital in Taipei in northern Taiwan. We enrolled all the patients

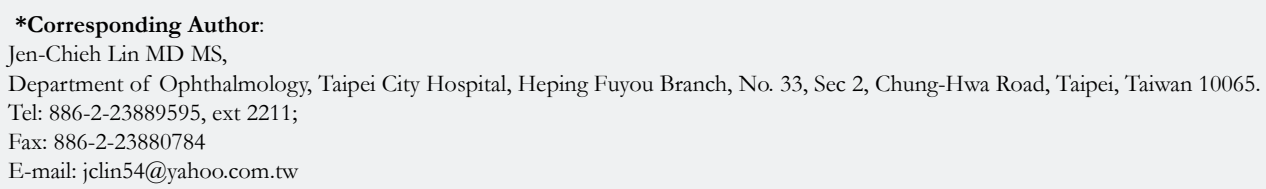

Citation: Hsieh MH, Lin JC (2016) Visual Field Loss, Quality of Life and Ocular Biometric Parameters: A Cross Sectional Study of Taiwanese Adults. Int J Ophthalmol Eye Res. 4(1), 165169. doi: http://dx.doi.org/10.19070/2332-290X-1600035

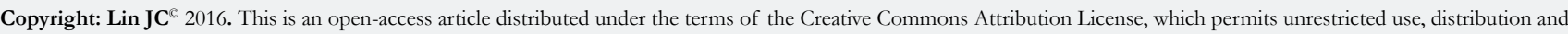
reproduction in any medium, provided the original author and source are credited. 
meeting our criteria in our outpatient department since July 2013 to June 2014. The subjects who did not accept the informed consent were excluded. This study received the approval of Institutional Review Board of the hospital and adhered to the tenets of the Declaration of Helsinki.

Detailed ocular examination was performed, including intraocular pressure, cycloplegic refractive error, optical coherence tomography, and FDT perimetry. A single interviewer administered the face-to-face questionnaire of Chinese (Taiwanese) version of NEIVFQ-25 to each participant.

The inclusion criteria were: adults between 20 and 50 years of age, the best corrected visual acuity $(\mathrm{BCVA})>0.8$ in the both eyes, without corneal diseases, cataract, or other retinal diseases. The patients were excluded if they had undergone intraocular surgery. The better eye of each subject was selected according to the abnormal scores on the FDT perimetry.

Ocular images were obtained in three modes of Spectraldomain optical coherence tomography (OCT Optovue RTVue, Inc Fremont, CA), including central cornea thickness (CCT), thickness of retinal nerve fiber layer (RNFL), and ganglion cell complex (GCC). The focal loss volume (FLV) and global loss volume (GLV) of GCC were also calculated.

FDT perimeter (Carl Zeiss Meditec, Dublin, Calif) using the N-30 protocol to evaluate visual field defects. Each participant had an additional test if any false-positive or false-negative error was recorded in the first test. In case acceptable results were not obtained, another visit would be arranged for the examination one month later. Visual field defects were determined by a FDT staging system [3], based on both the statistical significance and the spatial location of depressed points in the total deviation probability map. Accordingly, participants were classified into four groups as follows: (1) normal tests: score 0 to 1 , (2) early visual field loss (VFL): score 2 to 40, (3) moderate VFL: score 41 to 160 , and (4) severe VFL: score greater than 160.

Vision-related QoL was assessed by the NEI VFQ-25. This survey is composed of 25 items [4, 5]. Results were presented as a global score and 10 subscale scores, including general vision, ocular pain, near activities, distance activities, social function, mental health, role difficulties, dependency, color vision, and peripheral vision. Considering the potential effects of visual field loss on the near and distance activities, the global score and the subscales of near and distance activities were also included in the analysis.

\section{Statistical analysis}

Logistic regression models for the biometric factors and FDT staging were presented by coefficients $(\beta)$ in different groups. Linear regression models were applied to obtain weight coefficients $(\beta)$ with $95 \%$ confidence intervals (CI) for comparing the demographic, biometric factors, and parameters of FDT and VFQ. The Kruskal-Wallis method was used to analyze the difference in global and subscale scores of NEI VFQ-25 among different groups of FDT staging. A two-sided $p$ value $<0.05$ was considered statistically significant. All statistical analyses were performed using SPSS statistical software, version 16.0 (SPSS Inc., Chicago, IL, USA).

\section{Results}

A total of 206 subjects (male, 110) with complete examinations were included in the analysis (Table 1). According to FDT staging $72(35 \%)$ were classified as normal. There were 101, 27, and 6 subjects stratified as early, moderate, and severe VFL. Significant inter-group differences $(p<0.01)$ were noted for age, spherical equivalent refraction (SE), CCT, RNFL, and GCC.

In logistic regression models with the normal group used as reference, the presence of moderate VFL was significantly associated with lower CCT $(\beta=0.98 ; 95 \%$ CI, 0.96-0.99; $p<0.01)$, lower GCC $(\beta=0.92 ; 95 \%$ CI, 0.86-0.98; $p<0.01)$, higher GLV $(\beta$ $=1.20 ; 95 \% \mathrm{CI}, 1.10-1.30 ; p<0.01)$ and lower spherical equivalent refraction $(\beta=0.68 ; 95 \% \mathrm{CI}, 0.59-0.79 ; p<0.01$ ) (Table 2$)$.

In multivariate regression analysis, females $(p<0.01, \beta=-5.53)$ and people who had thinner RNFL $(p<0.01, \beta=-0.22)$ or lower $\operatorname{FLV}(p=0.03, \beta=-0.85)$ had lower scores in global score of NEI VFQ-25. Besides, females had lower scores in distance activities subscale $(p<0.01)$ (Table 3).

The Kruskal-Wallis analysis showed significant differences in global score $(p=0.03)$ and distance subscale score $(p=0.01)$ of NEI VFQ-25 among different groups of FDT staging (Figure1).

\section{Discussion}

Results of this study showed that visual field defects were detected by acceptable FDT test in about $65 \%$ adults in our clinic. However, the results were not sufficient to make a diagnosis for treatment, more clinical manifestations and other examinations are required for diagnosing and determining treatment approach.

The present study revealed that moderate visual field loss was associated with decreased QoL and distance activities; however, early visual field loss was not linked to QoL. According to this study, preventing the progression from early to moderate visual field loss may offer potential benefit to patient's subjective perception of QoL. In a longitudinal study by Ricardo et al., progression of VFL measured by either FDT or SAP could predict significant loss of QoL, but SAP was more sensitive in predicting change [6]. In addition, subjects had an older mean age $(65.8 \pm 12.3$ years $)$ in the study compared to the participants in the present study (32.6 \pm 10.3 years), but FDT mean deviations of better eyes were similar between two studies $(-2.5 \pm 4.1$ vs. $-2.4 \pm 2.9)$.

The participants of our study were younger than those in other studies reported on QoL. Based on the criteria defined in this study, patients with the presence of mild cataracts were excluded, so that our study subjects were more likely to be pathophysiologically homogenous. On the other hand, females appeared to have a lower subjective QoL (mean, female: 79, male: $84, p=0.03$, data was not shown); however, the finding should be confirmed in further study. As younger people may also respond better for details and act more deftly, the QoL might be therefore decreased easily since they tend to have a higher performance standard.

Some studies have shown the relationship between QoL and biometric parameters. Carolina et al. had found that each $1-\mu \mathrm{m}-$ 
Figure 1. The distribution of the score of NEI VFQ-25 (QoL), and the subscales of near activities and distance activities in different groups of visual field loss of FDT grading. Kruskal-Wallis and post-hoc analysis showed significantly decreased scores in moderate and severe VFL groups than in normal test group in QoL (1a) and subscale of distance activities (1c).
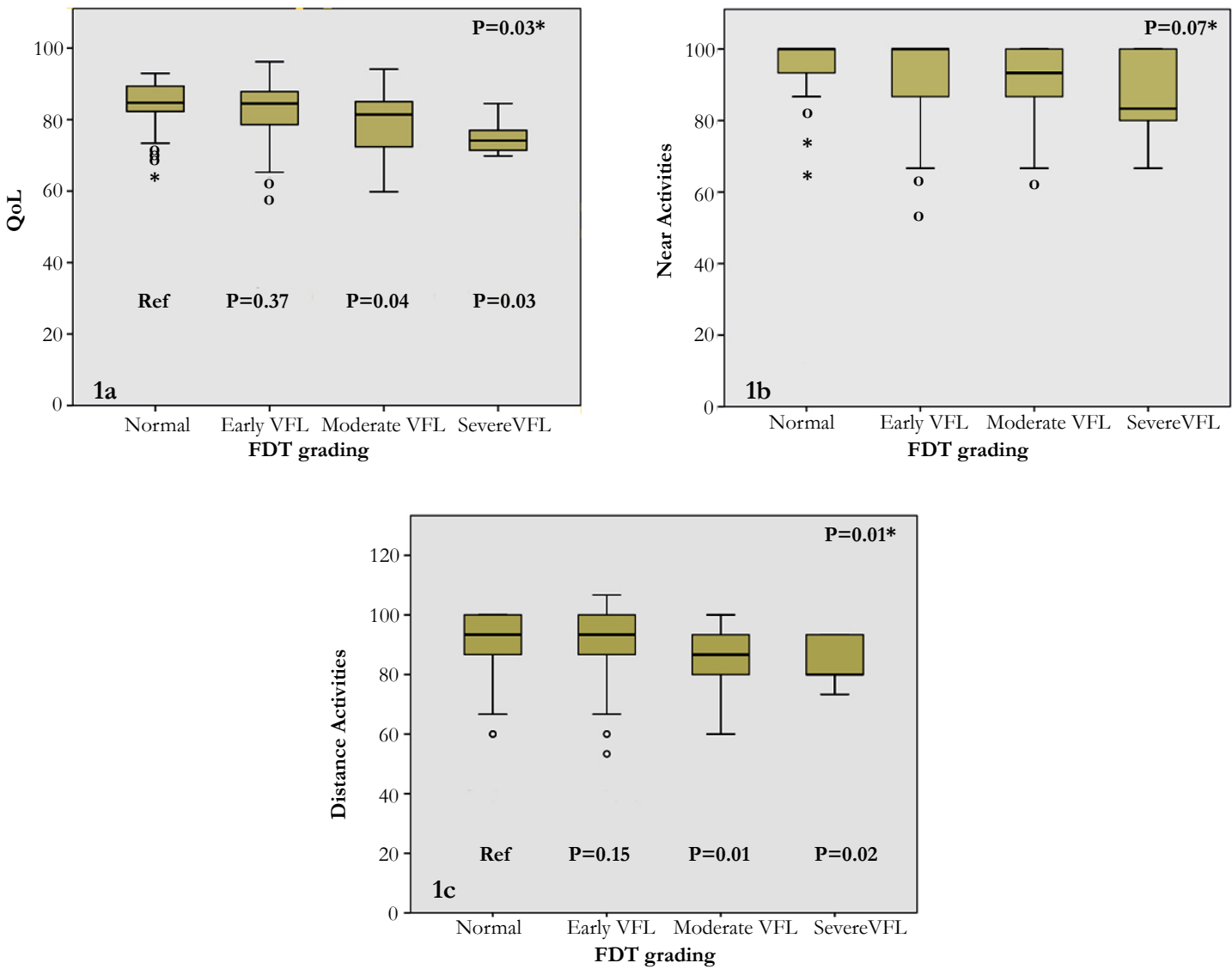

$*$ : p value of Kruskal-Wallis analysis

Abbreviation: VFL: visual field loss, QoL: quality of life, FDT: frequency doubling technology perimetry, Ref: reference

Table 1. Demographic and clinical characteristics of different groups of visual field defects.

\begin{tabular}{|c|c|c|c|c|c|c|c|c|c|c|}
\hline & \multirow{3}{*}{\multicolumn{2}{|c|}{\begin{tabular}{|c|} 
normal test \\
(score $0-1$ ) \\
\end{tabular}}} & \multirow{2}{*}{\multicolumn{2}{|c|}{$\begin{array}{c}\text { early defects } \\
\text { (score } 2-40)\end{array}$}} & \multirow{2}{*}{\multicolumn{2}{|c|}{\begin{tabular}{c|} 
moderate defects \\
(score $41-160)$
\end{tabular}}} & \multicolumn{2}{|c|}{ severe defects } & \multirow{4}{*}{$>$ p value* } & \multirow{3}{*}{ post hoc (scheffe) } \\
\hline & & & & & & & (score & & & \\
\hline & & $(n=72)$ & \multicolumn{2}{|c|}{$(\mathrm{n}=101)$} & \multicolumn{2}{|c|}{$(\mathrm{n}=27)$} & \multicolumn{2}{|c|}{$(n=6)$} & & \\
\hline & $\mathrm{N}$ & $\%$ & $\mathrm{~N}$ & $\%$ & $\mathrm{~N}$ & $\%$ & $\mathrm{~N}$ & $\%$ & & \\
\hline Gender & & & & & & & & & 0.66 & \\
\hline Male & 42 & 58.3 & 53 & 52.5 & 12 & 44.4 & 3 & 50.0 & & \\
\hline \multirow[t]{2}{*}{ Female } & 30 & 41.7 & 48 & 47.5 & 15 & 55.6 & 3 & 50.0 & & \\
\hline & \multicolumn{2}{|c|}{ Mean \pm SD } & \multicolumn{2}{|c|}{ Mean \pm SD } & \multicolumn{2}{|c|}{ Mean \pm SD } & \multicolumn{2}{|c|}{ Mean \pm SD } & & \\
\hline Age (years) & \multicolumn{2}{|c|}{$31.4 \pm 9.7$} & \multicolumn{2}{|c|}{$31.8 \pm 10.1$} & \multicolumn{2}{|c|}{$37.0 \pm 11.1$} & \multicolumn{2}{|c|}{$41.0 \pm 11.3$} & $<0.01$ & none \\
\hline SE (D) & \multicolumn{2}{|c|}{$-5.7 \pm 3.2$} & \multicolumn{2}{|c|}{$-7.1 \pm 3.7$} & \multicolumn{2}{|c|}{$-10.1 \pm 2.9$} & \multicolumn{2}{|c|}{$-10.6 \pm 4.3$} & $<0.01$ & $0>2,0>3,1>2,1>3 \dagger$ \\
\hline IOP $(\mathrm{mmHg})$ & \multicolumn{2}{|c|}{$16.9 \pm 3.5$} & \multicolumn{2}{|c|}{$16.4 \pm 3.5$} & \multicolumn{2}{|c|}{$14.6 \pm 3.3$} & \multicolumn{2}{|c|}{$16.7 \pm 4.3$} & 0.03 & $0>2 \dagger$ \\
\hline $\mathrm{CCT}(\mu \mathrm{m})$ & \multicolumn{2}{|c|}{$571.8 \pm 31.6$} & \multicolumn{2}{|c|}{$558.7 \pm 32.7$} & \multicolumn{2}{|c|}{$549.0 \pm 32.0$} & \multicolumn{2}{|c|}{$571.5 \pm 28.0$} & $<0.01$ & $0>1,0>2 \dagger$ \\
\hline $\mathrm{MD}(\mathrm{dB})$ & \multicolumn{2}{|c|}{$-0.2 \pm 1.4$} & \multicolumn{2}{|c|}{$-2.4 \pm 1.5$} & \multicolumn{2}{|c|}{$-6.6 \pm 2.2$} & -10.5 & & $<0.01$ & $0>1>2>3 \dagger$ \\
\hline PSD (dB) & $3.6=$ & 0.7 & 4.1 & & 6.6 & & $7.1 \pm$ & & $<0.01$ & $0<2,0<3,1<2,1<3 \dagger$ \\
\hline RNFL & 104.7 & $=9.2$ & 101.7 & 11.7 & 91.3 & & $81.8 \pm$ & & $<0.01$ & $0<2,0<3,1<2,1<3 \dagger$ \\
\hline GCC & 93.3 & 5.3 & 93.1 & 5.8 & 87.1 & & $81.1 \pm$ & & $<0.01$ & $0<2,0<3,1<2,1<3 \dagger$ \\
\hline FLV $(\%)$ & $0.9=$ & 1.3 & 1.4 & & 5.3 & & $6.1 \pm$ & & $<0.01$ & $0<2,0<3,1<2,1<3 \dagger$ \\
\hline GLV $(\%)$ & $5.7=$ & 3.9 & 6.1 & & 13.8 & & $19.3 \pm$ & & $<0.01$ & $0<2,0<3,1<2,1<3 \dagger$ \\
\hline
\end{tabular}

Abbreviations: SE, spherical equivalent refraction; IOP, intraocular pressure; CCT, central cornea thickness; MD, mean deviation; PSD, pattern standard deviation; RNFL, retinal nerve fiber layer; GCC, ganglion cell complex; FLV, focal volume loss; GLV, global volume loss.

* $\mathrm{p}$ value based on chi-squared tests or one way analysis of variance, comparing different levels of visual defects † 0 , normal test; 1 , early defects; 2 , moderate defects; 3 , severe defects 
Table 2. Logistic regression models of severity of visual defects according to parameters of clinical characteristics adjusted by gender and age.

\begin{tabular}{|c|c|c|c|c|c|c|c|c|c|}
\hline normal test & \multicolumn{3}{|c|}{ early VFL } & \multicolumn{3}{c|}{ moderate VFL } & \multicolumn{3}{c|}{ severe VFL } \\
\hline (score 0-1) & \multicolumn{3}{|c|}{ (score 2-40) } & \multicolumn{3}{c|}{ (score 41-160) } & \multicolumn{3}{c|}{ (score $>161)$} \\
\hline$(\mathrm{n}=72)$ & \multicolumn{3}{|c|}{$(\mathrm{n}=101)$} & \multicolumn{3}{c|}{$(\mathrm{n}=27)$} & \multicolumn{3}{c|}{$(\mathrm{n}=6)$} \\
\hline & $\beta$ & $95 \% \mathrm{CI}$ & $\mathrm{P}$ & $\beta$ & $95 \% \mathrm{CI}$ & $\mathrm{P}$ & $\beta$ & $95 \% \mathrm{CI}$ & $\mathrm{P}$ \\
\hline CCT & 0.98 & $0.97,0.99$ & $<0.01$ & 0.98 & $0.96,0.99$ & $<0.01$ & 1.01 & $0.98,1.04$ & 0.55 \\
\hline GLV & 1.02 & $0.95,1.09$ & 0.61 & 1.2 & $1.10,1.30$ & $<0.01$ & 1.26 & $1.10,1.44$ & $<0.01$ \\
\hline GCC & 0.99 & $0.95,1.04$ & 0.86 & 0.92 & $0.86,0.98$ & $<0.01$ & 0.86 & $0.76,0.97$ & 0.02 \\
\hline SE & 0.88 & $0.80,0.97$ & $<0.01$ & 0.68 & $0.59,0.79$ & $<0.01$ & 0.63 & $0.48,0.83$ & $<0.01$ \\
\hline
\end{tabular}

Abbreviations: CCT, central cornea thickness; GCC, ganglion cell complex; GLV, global loss volume; SE, spherical equivalent refraction; VFL, visual field loss.

Table 3. Multivariate linear regression analysis of the association between global score, near activities, and distance activities of vision related quality of life and demographic and ocular parameters.

\begin{tabular}{|c|c|c|c|c|c|c|c|c|c|}
\hline & \multicolumn{3}{|c|}{ Global score } & \multicolumn{3}{c|}{ Near activities } & \multicolumn{3}{c|}{ Distance activities } \\
\cline { 2 - 11 } & p value & $\beta$ & $95 \%$ CI of $\beta$ & p value & $\beta$ & $95 \%$ CI of $\beta$ & p value & $\beta$ & $95 \%$ CI of $\beta$ \\
\hline Male/Female & $<0.01$ & -5.53 & $-8.05,-3.01$ & 0.75 & -0.58 & $-4.11,2.96$ & $<0.01$ & -8.88 & $-12.34,-5.44$ \\
\hline Age (years) & 0.08 & 0.12 & $-0.01,0.26$ & $<0.01$ & -0.33 & $-0.52,-0.14$ & 0.02 & 0.22 & $0.04,0.40$ \\
\hline SE $(\mathrm{D})$ & 0.42 & 0.14 & $-0.20,0.46$ & 0.39 & -0.2 & $-0.66,0.26$ & 0.42 & 0.19 & $-0.27,0.64$ \\
\hline IOP $(\mathrm{mmHg})$ & 0.36 & -0.17 & $-0.53,0.19$ & 0.1 & -0.42 & $-0.93,0.09$ & 0.51 & 0.16 & $-0.33,0.66$ \\
\hline CCT $(\mu \mathrm{m})$ & 0.17 & 0.03 & $-0.01,0.07$ & 0.06 & 0.06 & $-0.01,0.11$ & 0.32 & 0.03 & $-0.03,0.08$ \\
\hline RNFL & $<0.01$ & -0.22 & $-0.35,-0.92$ & 0.12 & -0.15 & $-0.33,0.04$ & 0.37 & -0.08 & $-0.26,0.10$ \\
\hline GCC & 0.07 & -0.21 & $-0.44,0.01$ & 0.31 & -0.02 & $-0.05,0.02$ & 0.79 & -0.01 & $-0.04,0.03$ \\
\hline FLV $(\%)$ & 0.03 & -0.85 & $-1.62,-0.08$ & 0.92 & 0.05 & $-1.02,1.13$ & 0.3 & -0.55 & $-1.60,0.50$ \\
\hline GLV $(\%)$ & 0.11 & -0.4 & $-0.90,0.10$ & 0.95 & 0.02 & $-0.50,0.53$ & 0.84 & -0.51 & $-0.56,0.45$ \\
\hline
\end{tabular}

Abbreviations: SE, spherical equivalent; IOP, intraocular pressure; CCT, central cornea thickness; RNFL, retinal nerve fiber layer; GCC, ganglion cell complex; FLV, focal loss volume; GLV, global loss volume

per-year loss of RNFL thickness was associated with a decrease of 1.3 units (95\% CI, 1.02-1.56) per year in NEI VFQ-25 scores $(p<0.001)$ [7]. This study demonstrated a similar finding for the association between global score of NEI VFQ-25 and RNFL, but not the relationship between other subscales of NEI VFQ-25 and RNFL.

Our results also revealed a significant relationship between myopia and visual field loss, showing that a decrease in myopia of one diopter correlated with 0.68 -fold decrease in moderate visual field; however, myopia was not associated with QoL. Qiu $\mathrm{M}$ et al. demonstrated that subjects with mild to severe myopia had 2.02-fold to 12.43-fold risk of having any visual field defect in FDT perimetry when compared with those with emmetropia [8]. Similar results showing the association between myopia and visual defect in FDT perimetry were reported by Brian Chon's study on a Korean population [9]. In contrast, some studies have indicated no relationship between myopia and visual field loss in populations of similar characteristics $[10,11]$.

In addition, it is noteworthy that our findings for the relationship between QoL and VFL were similar to some investigations on other Asian populations. For example, our previous study demonstrating the association between the MD of FDT perimetry and NEIVFQ-25 [12]. Nah YS et al. have also reported the similar association between MD of Humphrey perimetry and visual function index using the questionnaire developed by Steinberg et al. [13].

There were still some limitations in our study. First, this was a cross-sectional study, so that data interpretation was limited by wide inter-individual variability. In addition, QoL assessment was based on subjective perceptions, which may be also influenced by inter-individual variability. However, evidence of repeated FDT with similar results may provide more confidence in our conclusion. Second, all of our participants were between 20 and 50 years old in age. The results might not be generalized to the whole population. Third, there were relatively few patients with severe visual field loss in our study population. Compared to older participants, a younger population would be more sensitive to subjective QoL and may be more vulnerable to slight change in visual field loss. Therefore, the results should be interpretated with caution when compared to different populations.

\section{Conclusion}

Vision related QoL was associated with FDT detected moderate visual field loss, but not early visual field loss. Considering the impacts of early visual field loss on subjective QoL, Results of this study may provide helpful support in making decision for treatment. Further studies are warranted to determine the potential of visual field loss as a factor for predicting vision loss related QoL. 


\section{References}

[1]. Johnson CA, Samuels SJ (1997) Screening for glaucomatous visual field loss with frequency-doubling perimetry. Invest Ophthalmol Vis Sci 38(2): 413425 .

[2]. Meira-Freitas D, Tatham AJ, Lisboa R, Kuang TM, Zangwill LM, et al. (2014) Predicting progression of glaucoma from rates of frequency doubling technology perimetry change. Ophthalmology 121(2): 498-507.

[3]. Brusini P, Tosoni C (2003) Staging of functional damage in glaucoma using frequency doubling technology. J Glaucoma 12(5): 417-426.

[4]. Mangione CM, Lee PP, Gutierrez PR, Spritzer K, Berry S, et al. (2001) Development of the 25-item National Eye Institute Visual Function Questionnaire. Arch Ophthalmol 119(7): 1050-1058.

[5]. Lin JC, Chie WC (2010) Psychometric validation of the Taiwan Chinese version of the 25-Item National Eye Institute Visual Functioning Questionnaire. J Eval Clin Pract 16(3): 619-626.

[6]. Abe RY, Gracitelli CP, Diniz-Filho A, Zangwill LM, Weinreb RN, et al. (2015) Frequency Doubling Technology Perimetry and Changes in Quality of Life of Glaucoma Patients: A Longitudinal Study. Am J Ophthalmol
160(1): 114-122.e1.

[7]. Gracitelli CP, Abe RY, Tatham AJ, Rosen PN, Zangwill LM, et al. (2015) Association between progressive retinal nerve fiber layer loss and longitudinal change in quality of life in glaucoma. JAMA Ophthalmol 133(4): 384-390.

[8]. Qiu M, Wang SY, Singh K, Lin SC (2013) Association between myopia and glaucoma in the United States population. Invest Ophthalmol Vis Sci 54(1): 830-835.

[9]. Chon B, Qiu M, Lin SC (2013) Myopia and glaucoma in the South Korean population. Invest Ophthalmol Vis Sci 54(10): 6570-6577.

[10]. Nouri-Mahdavi K, Hoffman D, Coleman AL, Liu G, Li G, et al. (2004) Predictive factors for glaucomatous visual field progression in the Advanced Glaucoma Intervention Study. Ophthalmology 111(9): 1627-1635.

[11]. Hung KH, Cheng CY, Liu CJ (2015) Risk factors for predicting visual field progression in Chinese patients with primary open-angle glaucoma: A retrospective study. J Chin Med Assoc 78(7): 418-423.

[12]. Lin JC, Yu JH (2012) Assessment of quality of life among Taiwanese patients with visual impairment. J Formos Med Assoc 111(10): 572-579.

[13]. Nah YS, Seong GJ, Kim CY (2002) Visual function and quality of life in Korean patients with glaucoma. Korean J Ophthalmol 16(2): 70-74. 